\title{
Rationale, study design and implementation of the COLM study: the combination of OLMesartan and calcium channel blocker or diuretic in high-risk elderly hypertensive patients
}

\author{
Toshio Ogihara ${ }^{1}$, Takao Saruta ${ }^{2}$, Hiromi Rakugi ${ }^{1}$, Kazuaki Shimamoto ${ }^{3}$, Sadayoshi Ito $^{4}$, Hiroaki Matsuoka ${ }^{5}$, \\ Masatsugu Horiuchi ${ }^{6}$, Tsutomu Imaizumi ${ }^{7}$, Shuichi Takishita ${ }^{8}$, Jitsuo Higaki ${ }^{6}$, Shigehiro Katayama ${ }^{9}$, \\ Ikuo Saito ${ }^{2}$ and Kazuyuki Shimada ${ }^{10}$, on behalf of the COLM study investigators
}

The COLM study is an investigator-initiated trial comparing the combination therapy using an angiotensin II receptor blocker (ARB), olmesartan, and a calcium channel blocker (CCB) with that using an ARB and a diuretic in high-risk elderly hypertensive patients. Here we describe the rationale and study design. Olmesartan was administered concomitantly with a long-acting dihydropyridine CCB (ARB/CCB group) or with a low-dose diuretic (ARB/diuretic group) to elderly hypertensive patients with a history of or risk factors for cardiovascular disease. Cardiovascular morbidity and mortality as a primary end point were compared between the two groups, with the target blood pressure (BP) being $<140 \mathrm{~mm} \mathrm{Hg}$ for systolic BP and $<90 \mathrm{~mm} \mathrm{Hg}$ for diastolic

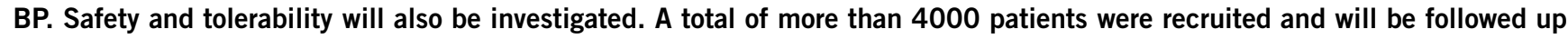
for at least 3 years.

Hypertension Research (2009) 32, 163-167; doi:10.1038/hr.2008.31; published online 23 January 2009

Keywords: clinical trial; combination therapy; olmesartan

\section{INTRODUCTION}

Hypertension is a key risk factor for cardiovascular disease. ${ }^{1}$ The main objective of treating hypertension is to reduce cardiovascular morbidity and mortality by decreasing high blood pressure (BP), as supported by the results of many large clinical trials and meta-analyses. ${ }^{2-6}$ According to various guidelines on the treatment of hypertension, the target BP for elderly hypertensive patients is $<140 / 90 \mathrm{~mm} \mathrm{Hg} .{ }^{4-6}$ It is not easy to achieve this target and it is often necessary to combine antihypertensive agents with different mechanisms of action. Combination therapy may reduce adverse drug reactions compared with monotherapy. For example, the incidence of hypokalemia due to diuretics may be reduced by combination with renin-angiotensin inhibitors. ${ }^{7}$

For antihypertensive therapy in elderly patients, the Japanese Society of Hypertension Guidelines for the Management of Hypertension (JSH 2004) recommend calcium channel blockers (CCBs), angiotensin II receptor blockers (ARBs), angiotensin-converting enzyme inhibitors (ACE inhibitors) and low-dose diuretics as the first-line drugs, and these agents should be used concomitantly if the target BP is not achieved. ARBs are commonly used to treat elderly hypertensive patients in Japan, ${ }^{8}$ and combination therapy with an $\mathrm{ARB}$ and a CCB or an ARB and a low-dose diuretic is recommended for hypertension. Evidence to support the benefit of such combination therapy on cardiovascular events has not been reported sufficiently.

We therefore planned to conduct the COLM (Combination of OLMesartan and calcium channel blocker or diuretic in high-risk elderly hypertensive patients) study, aiming to compare the combination of an ARB and a CCB with that of an ARB and a diuretic in the treatment of elderly hypertensive patients.

In this study, we selected olmesartan medoxomil for an ARB because it shows a good antihypertensive effect ${ }^{9-12}$ and is widely prescribed in both Japan and other countries.

\section{METHODS}

The study will be conducted using the prospective randomized open-blinded end point method. ${ }^{13}$ Participants will be randomized into two groups: one

${ }^{1}$ Osaka University Graduate School of Medicine, Osaka, Japan; ${ }^{2}$ Keio University, Osaka, Japan; ${ }^{3}$ Sapporo Medical University School of Medicine, Osaka, Japan; ${ }^{4}$ Tohoku University School of Medicine, Osaka, Japan; ${ }^{5}$ Dokkyo Medical University, Osaka, Japan; ${ }^{6}$ Ehime University Graduate School of Medicine, Osaka, Japan; ${ }^{7}$ Kurume University School of Medicine, Osaka, Japan; ${ }^{8}$ University of the Ryukyus School of Medicine, Osaka, Japan; ${ }^{9}$ Saitama Medical University, Osaka, Japan and ${ }^{10}$ Jichi Medical University, Osaka, Japan

Correspondence: Professor H Rakugi, Department of Geriatric Medicine and Nephrology, Osaka University Graduate School of Medicine, 2-2 Yamada-oka, Suita, Osaka 565-0871, Japan.

E-mail: rakugi@geriat.med.osaka-u.ac.jp

Received 14 September 2008; revised 31 October 2008; accepted 30 November 2008; published online 23 January 2009 
group that will receive combination treatment with olmesartan and a longacting dihydropyridine $\mathrm{CCB}$, and the other group that will receive olmesartan combined with a low-dose diuretic. Participants were recruited from April 2007 until September 2008 and will be followed up for at least 3 years (Figure 1).

\section{Enrollment of patients}

Hypertensive patients aged $\geqslant 65$ years and $<85$ years with a history of cardiovascular disease or risk factors for cardiovascular disease, including diabetes mellitus or dyslipidemia, were considered eligible. The investigator obtained written informed consent from the patients before their participation in the study. Patients who did not respond sufficiently to their prior antihypertensive therapy were enrolled and were randomly assigned to either group. Whether a patient met all the inclusion and exclusion criteria was checked after obtaining consent. For the details of the inclusion and exclusion criteria, refer to Appendix 1.

\section{Randomization}

The COLM Study Data Center randomized eligible patients to either the ARB/ $\mathrm{CCB}$ group or the $\mathrm{ARB} /$ diuretic group by a dynamic allocation method, in which sex, age ( $\geqslant 75$ years/ $<75$ years), history of cardiovascular disease, BP (mild/moderate or severe hypertension according to the JSH 2004 classification), prior use of antihypertensive agents and medical institution were used as factors for grouping.

\section{Study protocol}

The long-acting dihydropyridine CCBs and the low-dose diuretics used concomitantly with olmesartan are shown in Table 1. Azelnidipine and amlodipine are used as the CCBs, and thiazides are used as the diuretics. The initial doses of the drugs administered are as per the investigator's judgment. There are no restrictions on concomitant drugs apart from antihypertensive agents.

The target $\mathrm{BP}$ during the treatment period is set at $<140 \mathrm{~mm} \mathrm{Hg}$ for systolic $\mathrm{BP}$ (SBP) and at $<90 \mathrm{~mm} \mathrm{Hg}$ for diastolic BP (DBP) in both treatment arms. If the target $\mathrm{BP}$ is not achieved, the dose of olmesartan, $\mathrm{CCB}$ or diuretic will be increased. However, to prevent adverse events from increasing with higher doses of the study drugs, especially with an increase in dose of diuretic, the maximum doses are specified and are shown in Table 1. If the target BP is not achieved by the maximum dose of these basal drugs, other antihypertensive drugs, including $\beta$-blockers, $\alpha$-blockers and ACE inhibitors, will be added. If the BP decreases excessively, as judged by the participating physicians, antihypertensive agents other than the study drugs will be reduced or discontinued (if they are being used). If the BP is still too low, the dose of one of the study drugs will be reduced and combination therapy will be continued for as long as possible.

\section{Follow-up and laboratory tests}

The specified laboratory tests, investigation of the occurrence of cardiovascular events and measurement of BP will be conducted at 1,3 and 6 months after randomization, and then every 6 months. The laboratory tests will be conducted at each participating institution, and the investigator at each institution will report the results to the COLM Study Data Center. If a cardiovascular event occurs, it will be immediately reported to the COLM Study Data Center. If adverse events occur, appropriate treatment will be provided immediately. If the investigator judges that a participant with adverse events should discontinue the study, this will be done.

\section{Monitoring of safety}

Interim analyses will be conducted twice, in September 2009 and 2010, to decide whether to continue or discontinue the study based on ethical and scientific considerations. The Independent Data Monitoring Committee will analyze data on safety and cardiovascular events. If the Independent Data Monitoring Committee judges that continuation of the study is ethically or scientifically problematic, the committee will ask the Steering Committee to amend the study protocol or discontinue the study.

\section{End points}

The primary and secondary end points are shown in Table 2. Cardiovascular events reported by the participating physicians will be assessed by the Endpoint

Table 1 Combination antihypertensive treatments

\begin{tabular}{lll}
\hline & $\begin{array}{c}\text { Base antihypertensive drug: ARB } \\
\text { Olmesartan: 5-40 mg }\end{array}$ \\
\hline \multicolumn{3}{c}{ Concomitant antihypertensive drug } \\
Dihydropyridine CCB & \multicolumn{1}{c}{ Thiazide diuretic } \\
\hline Azelnidipine (8-16 mg) & Hydrochlorothiazide & $\leqslant 12.5 \mathrm{mg}$ \\
Amlodipine (2.5-5 mg) & Trichloromethiazide & $\leqslant 1 \mathrm{mg}$ \\
& Benzylhydrochlorothiazide & $\leqslant 2 \mathrm{mg}$ \\
& Indapamide & $\leqslant 1 \mathrm{mg}$ \\
& Meticrane & $\leqslant 75 \mathrm{mg}$ \\
& Tripamide & $\leqslant 25 \mathrm{mg}$ \\
& Chlorthalidone & $\leqslant 12.5 \mathrm{mg}$ \\
\hline
\end{tabular}

Abbreviations: ARB, angiotensin II receptor blocker; CCB, calcium channel blocker.

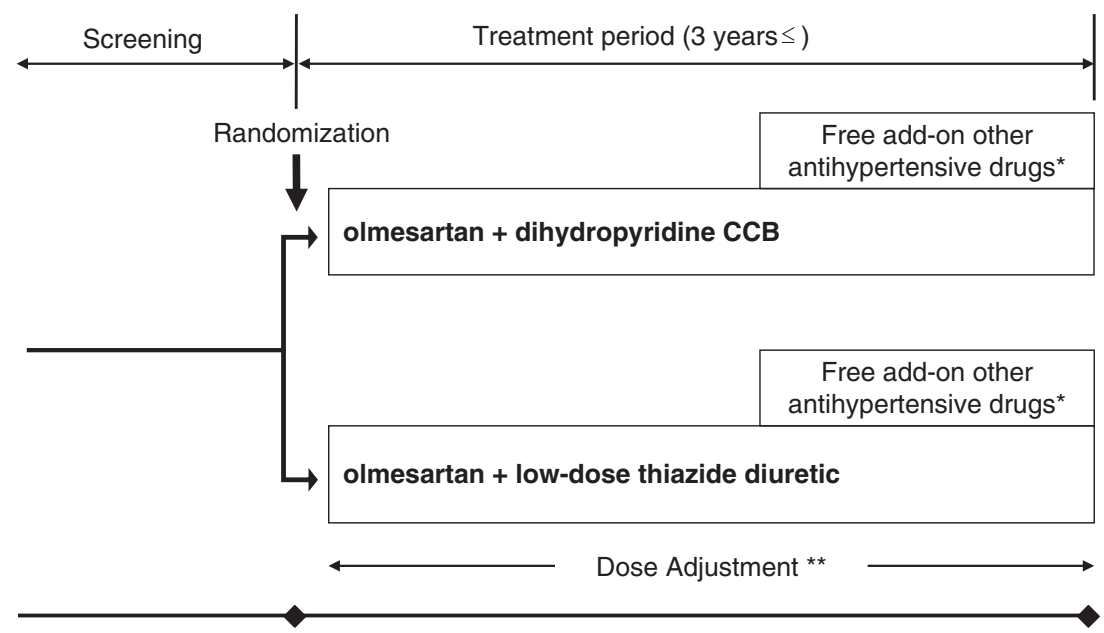

Figure 1 Study design of COLM study. CCB, calcium channel blocker. * $\beta$-blocker, $\alpha$-blocker or ACE inhibitor; **target BP: <140/90 mm Hg. 


\section{Primary end point}

A composite of fatal and non-fatal cardiovascular events

1. Sudden death (death of endogenous origin within $24 \mathrm{~h}$ after acute onset)

2. Cerebrovascular events (new occurrence or recurrence of cerebral infarction, cerebral hemorrhage, subarachnoid hemorrhage or transient ischemic attack)

3. Cardiac events (new occurrence or recurrence of myocardial infarction, coronary revascularization (PCI or CABG), hospitalization for angina pectoris or hospitalization for heart failure)

4. Renal dysfunction (doubling of serum creatinine and creatinine $\geqslant 2.0 \mathrm{mg}$ per $100 \mathrm{ml}$, ESRD)

Secondary end points

1. Composite of hard endpoint: cardiovascular death, non-fatal myocardial infarction, non-fatal stroke except transient ischemic attack

2. All deaths

3. Death from cardiovascular events

4. Effects on glucose metabolism (fasting plasma glucose, postprandial glucose, new onset of diabetes mellitus)

5. Incidence of subset of primary endpoint

5-1. Sudden death

5-2. Cerebrovascular events

5-3. Cardiac events

5-4. Renal dysfunction

6. New occurrence of atrial fibrillation

7. Safety

8. Proportion of the subjects who withdrew from the allocated treatment

Abbreviations: CABG, coronary artery bypass graft; ESRD, end-stage renal disease; $\mathrm{PCl}$, percutaneous coronary intervention.

Committee on a blinded basis in accordance with the cardiovascular event assessment criteria.

\section{Statistical analysis}

Sample size. The annual incidence of cardiovascular events in elderly Japanese patients treated with CCBs or ACE inhibitors is reported to be $2-3 \%\left(20-30 / 1000\right.$ patients). ${ }^{14}$ If the incidence of cardiovascular events (the primary end point) is $2 \%$ per year and the relative difference in the incidence of cardiovascular events between the two groups is assumed to be 33\%, then 1999 participants per group would be required to achieve $\alpha=0.05$ (two sided) and a power of $80 \%$. On the basis of this calculation, more than 2000 participants will be randomized to each group.

\section{Statistical analysis plan}

Major analyses will be conducted according to the intention-to-treat principle. For major analyses, the frequency of the primary and secondary end points will be compared between the ARB/CCB group and the ARB/diuretic group. For the intergroup comparison of cardiovascular events, a stratified log-rank test will be conducted using sex, age and history of cerebrovascular/cardiovascular disease as stratification variables. Time-to-event curves for cardiovascular events in each group will be estimated by the Kaplan-Meier method. The hazard ratio and its 95\% confidence interval will be calculated by using a stratified Cox proportional hazards model.

Prespecified subgroup analyses will be conducted as exploratory analyses and interactions between factors in each group and subgroup will be investigated.

For all analyses, overall, the level of significance will be $P=0.05$, and two-sided tests will be used. The details will be described in the statistical analysis plan.

\section{Interim analysis}

To judge whether to continue or discontinue the study for ethical reasons, two interim analyses will be conducted. The results will be submitted to the Independent Data Monitoring Committee. On the basis of the findings, the committee may request discontinuation of the study or improvement of treatment compliance.

Early discontinuation of the study based on the results of interim analysis will depend on the primary end point. Multiplicity of testing in the interim analysis of the primary end point will be adjusted using the Lan-DeMets alphaspending function, ${ }^{15}$ approximating an O'Brien-Fleming boundary. ${ }^{16}$

\section{Data management}

Case report forms filled out by the investigators will be collected by the COLM Study Data Center. The COLM Study Data Center will check compliance with the protocol as necessary and will notify the investigators of requirements for maintaining the quality of the study. The investigators will keep source documents for essential data (laboratory tests, investigational items, etc.). The COLM Study Data Center will conduct centralized monitoring of the participating institutions, visit as necessary, and check the source documents and information entered by the investigators.

\section{Institutional review board}

Before starting the study, the central institutional review board (IRB) will review the protocol on behalf of the principal researchers from the ethical and safety viewpoints, and will also assess the informed consent form. Participation in the study is approved by the IRB of each medical institution. If the IRB is not established at a particular medical institution, site participation is approved by the central IRB as requested by the principal researchers.

\section{DISCUSSION}

The objective of treating hypertension is to prevent cardiovascular disease, including damage to the brain, heart, kidneys and blood vessels associated with hypertension, as well as death from cardiovascular disease. Especially in patients who have risk factors for cardiovascular disease, it is important to achieve the target BP for both primary and secondary prevention of cardiovascular disease. ${ }^{3}$ To achieve the target BP, combination therapy involving multiple antihypertensive agents with different mechanisms of action is a useful strategy. JNC7 recommends starting treatment for stage 2 (SBP $\geqslant 160$ or $\mathrm{DBP} \geqslant 100 \mathrm{~mm} \mathrm{Hg}$ ) hypertensive patients with combination therapy. The 2007 European Society of Hypertension/European Society of Cardiology Guidelines recommend starting treatment with a two-drug combination at low dose for patients with marked BP elevation or high cardiovascular risk (ESH-ESC2007). In hypertensive patients whose BP could not be controlled by the maximum dose of an 
$\mathrm{ARB}$, adding a diuretic or $\mathrm{CCB}$ was reported to enhance the antihypertensive effect. ${ }^{17,18}$

The ultimate goal of combination therapy is to reduce cardiovascular outcomes in addition to reducing BP. Only two large-scale randomized clinical trials have investigated which combination of antihypertensive agents is more effective in preventing cardiovascular diseases in hypertensive patients. One is ASCOT-BPLA (Anglo-Scandinavian Cardiac Outcomes Trial-Blood Pressure Lowering ARM) ${ }^{19}$ and the other is ACCOMPLISH (Avoiding Cardiovascular Events Through COMbination Therapy in Patients LIving with Systolic Hypertension). ${ }^{20}$

The ASCOT-BPLA compared amlodipine-based therapy, adding perindopril as required, with atenolol-based therapy, adding bendroflumethiazide as required. Both protocols used combination therapy in more than half of the patients. ${ }^{19}$ The ASCOT-BPLA clearly revealed that amlodipine-based therapy was superior to atenolol-based therapy in reducing cardiovascular events. On the basis of this finding, combination therapy with a $\beta$-blocker and a diuretic was excluded from the list of preferable combinations in the 2007 European Society of Hypertension/European Society of Cardiology Guidelines.

ACCOMPLISH compared combination therapy using an ACE inhibitor and a CCB with that using an ACE inhibitor and a diuretic. ${ }^{20}$ The primary result of ACCOMPLISH showed the superiority of the ACE inhibitor-CCB combination. ${ }^{21}$

The COLM study has a similar design to the ACCOMPLISH study. However, there are several differences in the protocol. The first difference between the studies is the basal drug; an ARB is being used in the COLM study, whereas an ACE inhibitor was used in the ACCOMPLISH study. Both drugs are inhibitors of the renin-angiotensin system but have different mechanisms of action. Considering the side effects of ACE inhibitors, such as dry cough, ${ }^{22}$ a higher rate of study completion is expected in the COLM study compared with the ACCOPLISH study.

The second difference between the two studies is the dose of the concomitant diuretic. In the COLM study, diuretics are used at low doses only, whereas the ACCOMPLISH study allowed the diuretic dose to be gradually increased up to the usual level. When diuretics are used for combination therapy, low doses are generally employed considering both the antihypertensive effect and the metabolic effects. ${ }^{23}$ In the COLM study, low doses of diuretics are being used, and we expect to obtain results that can also be achieved in clinical practice.

The third difference between the two studies is the study population. The COLM study recruited Japanese elderly patients $(\geqslant 65$ years), whereas the ACCOPMLISH study recruited mainly Caucasians $(84 \%)$ aged $\geqslant 55$ years. Therefore, the study participants in the COLM study might be more salt sensitive than those in the ACCOMPLISH study.

The COLM study is being conducted using the prospective randomized open-blinded end point method, and a composite end point is being investigated. There are concerns about whether it is reasonable to assess the primary end point because an index that may bias the observation is included in the composite end point. ${ }^{24} \mathrm{We}$ also plan to analyze a composite of hard end point including 'cardiovascular death, non-fatal myocardial infarction and non-fatal stroke except transient ischemic attack' as a secondary end point and investigate whether it shows the same trend as the primary end point. This will be helpful to confirm the reasonableness of using a composite of fatal and non-fatal cardiovascular events as the primary end point.

A number of fixed-combination antihypertensive drugs have recently been launched in Japan and other countries, and there are many options available for the treatment of hypertension. We believe that the COLM study will provide useful information about combination antihypertensive therapy in elderly patients, which can prevent cardiovascular disease and promote good adherence during long-term treatment, and will assist the creation of guidelines for the selection of antihypertensive therapy and antihypertensive drugs, including combination drugs.

\section{ACKNOWLEDGEMENTS}

The Japan Heart Foundation has awarded a grant to assist in conducting the COLM study.

We would like to express our gratitude to the doctors participating in the COLM study.

1 Lewington S, Clarke R, Qizilbash N, Peto R, Collins R. Age-specific relevance of usual blood pressure to vascular mortality: a meta-analysis of individual data for one million adults in 61 prospective studies. Lancet 2002; 360: 1903-1913.

2 The ALLHAT Officers and Coordinators for the ALLHAT Collaborative Research Group. Major outcomes in high-risk hypertensive patients randomized to angiotensin-converting enzyme inhibitor or calcium channel blocker vs diuretic: The Antihypertensive and Lipid-Lowering treatment to prevent Heart Attack Trial (ALLHAT). JAMA 2002; 288: 2981-2997.

3 Staessen JA, Wang JG, Thijs L. Cardiovascular protection and blood pressure reduction: a meta-analysis. Lancet 2001; 358: 1305-1315.

4 Japanese Society of Hypertension. Japanese Society of Hypertension Guidelines for the Management of Hypertension (JSH 2004). Hypertens Res 2006; 29: S1-S105.

5 Chobanian AV, Bakris GL, Black HR, Cushman WC, Green LA, Izzo Jr JL, Jones DW, Materson BJ, Oparil S, Wright Jr JT, Roccella EJ, Joint National Committee on Prevention, Detection, Evaluation, Treatment of High Blood Pressure. National Heart, Lung, and Blood Institute; National High Blood Pressure Education Program Coordinating Committee. Seventh report of the Joint National Committee on Prevention, Detection, Evaluation, and Treatment of High Blood Pressure. Hypertension 2003; 42: $1206-1252$.

6 Mancia G, De Backer G, Dominiczak A, Cifkova R, Fagard R, Germano G, Grassi G, Heagerty AM, Kjeldsen SE, Laurent S, Narkiewicz K, Ruilope L, Rynkiewicz A, Schmieder RE, Boudier HA, Zanchetti A, Vahanian A, Camm J, De Caterina R, Dean V, Dickstein K, Filippatos G, Funck-Brentano C, Hellemans I, Kristensen SD, McGregor K, Sechtem U, Silber S, Tendera M, Widimsky P, Zamorano JL, Erdine S, Kiowski W, Agabiti-Rosei E, Ambrosioni E, Lindholm LH, Viigimaa M, Adamopoulos S, Agabiti-Rosei E, Ambrosioni E, Bertomeu V, Clement D, Erdine S, Farsang C, Gaita D, Lip G, Mallion JM, Manolis AJ, Nilsson PM, O'Brien E, Ponikowski P, Redon J, Ruschitzka F, Tamargo J, van Zwieten P, Waeber B, Williams B, , Management of Arterial Hypertension of the European Society of Hypertension; European Society of Cardiologylpar. 2007 Guidelines for the Management of Arterial Hypertension: The Task Force for the Management of Arterial Hypertension of the European Society of Hypertension(ESH) and of the European Society of Cardiology(ESC). J Hypertens 2007; 25: 1105-1187.

7 Sica DA. Rationale for fixed-dose combinations in the treatment of hypertension: the cycle repeats. Drugs 2002; 62: 443-462.

8 Kamijima Y, Ooba N, Yagame M, Samizo K, Shimodozono Y, Kageyama S, Horiguchi S, Nagai R, Kusunoki T, Kubota K. Hypertension management in diabetic patients: prescribing trends from 1999 to 2005 in three Japanese university hospitals. Pharmacoepidemiol Drug Saf 2008; 17: 904-911.

9 Brunner HR, Arakawa K. Antihypertensive efficacy of olmesartan medoxomil and candesartan cilexetil in achieving 24-h blood pressure reductions and ambulatory blood pressure goals. Clin Drug Investig 2006; 26: 185-193.

10 Oparil S, Williams D, Chrysant SG, Marbury TC, Neutel J. Comparative efficacy of olmesartan, losartan, valsartan, and irbesartan in the control of essential hypertension. J Clin Hypertens 2001; 3: 283-291, 318.

11 Saito I, Kushiro T, Hirata K, Sato Y, Kobayashi F, Sagawa K, Hiramatsu K, Komiya M. The use of olmesartan medoxomil as monotherapy or in combination with other antihypertensive agents in elderly hypertensive patients in Japan. J Clin Hypertens 2008; 10: 272-279.

12 Nakayama S, Watada H, Mita T, Ikeda F, Shimizu T, Uchino H, Fujitani Y, Hirose T, Kawamori R. Comparison of effects of olmesartan and telmisartan on blood pressure and metabolic parameters in Japanese early-stage type-2 diabetics with hypertension. Hypertens Res 2008; 31: 7-13.

13 Hansson L, Hedner T, Dahlöf B. Prospective randomized open blinded end-point (PROBE) study. A novel design for intervention trials. Prospective Randomized Open Blinded End-Point. Blood Press 1992; 1: 113-119.

14 Ogihara T. Practitioner's Trial on the Efficacy of Antihypertensive Treatment in the Elderly Hypertension (The PATE-Hypertension Study) in Japan. Am J Hypertens 2000; 13: 461-467. 
15 Lan KKG, DeMets DL. Discrete sequential boundaries for clinical trials. Biometrika 1983; 70: 659-663.

16 O'Brien PC, Fleming TR. A multiple testing procedure for clinical trials. Biometrics 1979; 35: 549-556.

17 Stergiou GS, Makris T, Papavasiliou M, Efstathiou S, Manolis A. Comparison of antihypertensive effects of an angiotensin-converting enzyme inhibitor, a calcium antagonist and a diuretic in patients with hypertension not controlled by angiotensin receptor blocker monotherapy. J Hypertens 2005; 23: 883-889.

18 Neutel JM, Smith DH, Silfani TN, Lee Y, Weber MA. Effects of a structured treatment algorithm on blood pressure goal rates in both stage 1 and stage 2 hypertension. J Hum Hypertens 2006; 20: 255-262.

19 Dahlöf B, Sever PS, Poulter NR, Wedel H, Beevers DG, Caulfield M, Collins R, Kjeldsen SE, Kristinsson A, McInnes GT, Mehlsen J, Nieminen M, O'Brien E, Ostergren J, ASCOT Investigators. Prevention of cardiovascular events with an antihypertensive regimen of amlodipine adding perindopril as required versus atenolol adding bendroflumethiazide as required, in the Anglo-Scandinavian Cardiac Outcomes Trial-Blood Pressure Lowering Arm (ASCOT-BPLA): a multicentre randomised controlled trial. Lancet 2005; 366: 895-906.

20 Jamerson KA, Bakris GL, Wun CC, Dahlöf B, Lefkowitz M, Manfreda S, Pitt B, Velazquez EJ, Weber MA. Rationale and design of the avoiding cardiovascular events through combination therapy in patients living with systolic hypertension (ACCOMPLISH) trial: the first randomized controlled trial to compare the clinical outcome effects of first-line combination therapies in hypertension. Am J Hypertens 2004; 17: 793-801.

21 Jamerson K, Weber MA, Bakris GL, Dahlöf B, Pitt B, Shi V, Hester A, Gupte J, Gatlin M, Velazquez EJ, for the Accomplish trial investigators. Benazepril plus Amlodipine or Hydrochlorothiazide for Hypertension in High-Risk Patients. N Engl J Med 2008; 359: 2417-2428.

22 Matchar DB, McCrory DC, Orlando LA, Patel MR, Patel UD, Patwardhan MB, Powers B, Samsa GP, Gray RN. Systematic Review: Comparative Effectiveness of AngiotensinConverting Enzyme Inhibitors and Angiotensin II Receptor Blockers for Treating Essential Hypertension. Ann Intern Med 2008; 148: 16-29.

23 Murai K, Obara T, Ohkubo T, Metoki H, Oikawa T, Inoue R, Komai R, Horikawa T, Asayama K, Kikuya M, Totsune K, Hashimoto J, Imai Y, J-Home Study Group. Current usage of diuretics among hypertensive patients in Japan: the Japan Home Versus Office blood Pressure Measurement Evaluation (J-HOME) Study. Hypertens Res 2006; 29: 857-863.

24 Freemantle N, Calvert M, Wood J, Eastaugh J, Griffin C. Composite outcomes in randomized trials: greater precision but with greater uncertainty? JAMA 2003; 289: 2554-2559.

\section{APPENDIX 1}

\section{Inclusion and exclusion criteria}

Inclusion criteria

- Age: 65-84 years.

- $\mathrm{SBP} \geqslant 140 \mathrm{~mm} \mathrm{Hg}$ or $\mathrm{DBP} \geqslant 90 \mathrm{~mm} \mathrm{Hg}$ in a sitting position on two consecutive measurements at the clinic during the use of one or more antihypertensive medications.

- $\mathrm{SBP} \geqslant 160 \mathrm{~mm} \mathrm{Hg}$ or $\mathrm{DBP} \geqslant 100 \mathrm{~mm} \mathrm{Hg}$ in a sitting position on two consecutive measurements at the clinic without antihypertensive medication.

Require at least one of the following medical history or risk factors.

\section{Medical history}

1. Cerebrovascular accident: cerebral infarction, cerebral hemorrhage or subarachnoid hemorrhage (6 months or more before registration).

2. Myocardial infarction or coronary revascularization (PCI or CABG) (6 months or more before registration).

3. Angina pectoris (except for the patients having a history of hospitalization within 6 months before registration).
Risk factors

1. Male.

2. Current diabetes mellitus, fasting glucose $\geqslant 110 \mathrm{mg}$ per $100 \mathrm{ml}$ or postprandial glucose $\geqslant 140 \mathrm{mg}$ per $100 \mathrm{ml}$

3. Hypercholesterolemia (total cholesterol $\geqslant 260 \mathrm{mg}$ per $100 \mathrm{ml}$ ).

4. Low HDL cholesterolemia (HDL-C $<40 \mathrm{mg}$ per $100 \mathrm{ml}$ ).

5. Microalbuminuria (urinary albumin creatinine ratio $\geqslant 30 \mathrm{mg} / \mathrm{gCr}$ ) or proteinuria (protein $\geqslant 1+$ ).

6. Left ventricular hypertrophy (ST-T change in the ECG and $\mathrm{SV} 1+\mathrm{RV} 5 \geqslant 35 \mathrm{~mm}$, or left ventricular mass index: male $\geqslant 125 \mathrm{~g} \mathrm{~m}^{-2}$, female $\geqslant 110 \mathrm{~g} \mathrm{~m}^{-2}$ ).

\section{Exclusion criteria}

1. Secondary hypertension or malignant hypertension.

2. History of cerebrovascular accident (including TIA) or myocardial infarction within 6 months before registration.

3. PCI or CABG performed within 6 months before registration or scheduled.

4. History of hospitalization for angina pectoris or heart failure within 6 months before registration.

5. Severe heart failure (New York Heart Association functional class III or more severe)

6. Atrial fibrillation, atrial flutter or severe arrhythmia.

7. Severe hepatic or renal dysfunction (including current treatment by dialysis or renal dysfunction with serum creatinine $\geqslant 2.0 \mathrm{mg}$ per $100 \mathrm{ml}$ ).

8. Not appropriate for change to the study drugs from current therapy for concurrent disease including coronary heart diseases (that is, calcium channel blockers, diuretics, etc.).

9. History of serious side effects from study drugs (ARB, calcium channel blocker or diuretic).

10. Life-threatening condition (malignant tumor, etc.).

11. Not suited to be a study participant as judged by a study physician.

\section{APPENDIX 2}

\section{Membership of committees}

Steering Committee: Takao Saruta (Chair), Toshio Ogihara (Chair), Kazuaki Shimamoto, Sadayoshi Ito, Hiroaki Matsuoka, Kazuyuki Shimada, Masatsugu Horiuchi, Tsutomu Imaizumi and Shuichi Takishita.

Protocol Committee: Kazuyuki Shimada, Nobuyuki Ura, Koichi Hayashi and Hiromi Rakugi.

End point Committee: Jitsuo Higaki, Masato Odawara, Norio Tanahashi and Genjiro Kimura.

Independent Data Monitoring Committee: Shigehiro Katayama, Satoshi Umemura, Toshihiko Ishimitsu, Naoki Kashihara and Satoshi Morita. Statistical Committee: Ikuo Saito and Satoshi Teramukai.

Ethics Committee: Masashi Kobayashi, Mariko Fujikawa, Yoshio Uetsuka, Ryo Nagao and Mitsuyo Ito.

Study Secretary: Hiromi Rakugi. 